Recebido em 02/2019. Aceito para publicação em 05/2019.

\title{
AVALIAÇÃO E PERCEPÇÃO DE SUSTENTABILIDADE AMBIENTAL EM UNIDADES PRODUTORAS DE REFEIÇÕES DE CLUBES PAULISTANOS
}

\section{EVALUATION AND PERCEPTION OF ENVIRONMENTAL SUSTAINABILITY IN FOOD SERVICES OF PAULISTAN CLUBS}

\author{
Ingrid Rezende França ${ }^{1}$ \\ Mônica Glória Neumann Spinelli ${ }^{2}$ \\ Juliana Masami Morimoto ${ }^{3}$
}

Resumo: A produção de refeições envolve processos que causam impactos ambientais. A melhora desses fatores é um desafio para os gestores que precisam conciliar esses aspectos aos custos e necessidades dos clientes. O objetivo do estudo foi avaliar a sustentabilidade ambiental em unidades produtoras de refeições e a percepção do nutricionista neste quesito. Foi aplicado um "check list" para obtenção de aspectos de sustentabilidade e um questionário para avaliação da percepção do nutricionista, ambos com pontuação que equivalem até $100 \%$. Os dados foram analisados por meio da média e desvio padrão. Foi utilizado o teste $t$ de Student para analisar a diferença entre duas médias e análise de variância (ANOVA) para analisar a diferença entre três ou mais médias. As análises de associação entre duas variáveis quantitativas foram realizadas por meio da correlação de Pearson. Todos os testes estatísticos consideraram nível de significância de 5\%. Do total de restaurantes ( $n=7), 71,4 \%$ $(n=5)$ possuíam programas voltados para sustentabilidade ambiental. Apenas um (14,3\%) obteve pontuação de $77,4 \%$ no "check list" de sustentabilidade, sendo classificado como "bom" e 85,7\% (n=6) apresentaram pontuação de 50 a 74\%, com classificação de "aceitável". Os locais que dispunham de programas voltados para sustentabilidade apresentaram pontuações mais elevadas. Obteve-se correlação fraca ao analisar a associação entre a percepção de sustentabilidade e a pontuação do "check list". Foi possível verificar que a maioria dos nutricionistas possuem elevada percepção sobre sustentabilidade, porém ao observar as pontuações do "check list", percebe-se que são poucas as ações realizadas de modo a minimizar o impacto ambiental.

Palavras-chave: Impacto ambiental; nutricionistas; restaurantes; sustentabilidade.

Abstract: Meal production involves processes that cause environmental impacts. Improving these factors is a challenge for managers who need to reconcile these aspects with clients' costs and needs. The objective of the study was to evaluate the environmental sustainability in food services and the nutritionist's perception in this regard. A check list was applied to obtain sustainability aspects and a questionnaire to evaluate the perception of the nutritionist, both with a score of up to $100 \%$. The data were analyzed by mean and standard deviation. Student's t-test was used to analyze the difference between two means and analysis of variance (ANOVA) to analyze the difference among three or more means. The analyses of association between two quantitative variables were performed using the Pearson's correlation. All statistical tests considered a significance level of $5 \%$. Of the total of restaurants $(n=7), 71.4 \%(n=5)$ had programs focused on environmental sustainability. Only one (14.3\%) had a score of $77.4 \%$ on the sustainability check list, being classified as "good" and $85.7 \%(n=6)$ presented a score of 50 to $74 \%$, with a score of "acceptable ". Places with sustainability-focused programs had higher scores. A weak correlation was obtained when analyzing the association between the perception of sustainability and the check list score. It was possible to verify that most nutritionists have a high perception about sustainability, but observing the check list scores, it is noticed that few actions are taken in order to minimize the

\footnotetext{
1 Graduado em Nutrição, Universidade Presbiteriana Mackenzie, Brasil. E-mail: ingrid3.franca@gmail.com.

2 Doutora em Saúde Pública pela Universidade de São Paulo, Docente no curso de Nutrição, Universidade Presbiteriana Mackenzie, Brasil. E-mail: monica.spinelli@mackenzie.br.

3 Doutora em Saúde Pública pela Universidade de São Paulo, Docente no curso de Nutrição, Universidade Presbiteriana Mackenzie, Brasil. E-mail: juliana.morimoto@mackenzie.br.
} 
environmental impact.

Keywords: Environmental impact; nutritionists; restaurants; sustainability.

\section{INTRODUÇÂO}

No modo de vida urbano atual, a indústria e os serviços de alimentos propiciam ao consumidor uma infraestrutura cuja lógica é pautada pela otimização do tempo e trabalho, pois a pressa é um dos traços mais visíveis nas grandes cidades, com abreviamento do ritual alimentar em suas diferentes fases: da preparação ao consumo. $\mathrm{O}$ ato de comer transforma-se em uma simples operação de reabastecimento (DIEZ GARCIA, 2003). Por conseguinte, ocorre uma elevação do consumo de alimentos fora da residência, que pode ser observado através do aumento do percentual de gastos com alimentação fora do domicílio (IBGE, 2011).

A produção de refeições para coletividades acontece em locais específicos que podem ser chamados de Unidade Produtoras de Refeições (UPRs), Unidade de Alimentação e Nutrição (UAN) ou Serviço de Alimentação Coletiva (SAC). São utilizados também termos como Unidades de Alimentação (UA), serviço de restauração e mercado de food service (RODGERS, 2011). Essa demanda por refeições de consumo imediato colaborou para o crescimento do número de Unidades Produtoras de Refeições (UPRs) e trouxe diversidade a seus serviços (BEZERRA et al., 2017).

A finalidade desses locais é administrar a produção de refeições equilibradas em nutrientes, com bom padrão higiênico-sanitário, que possam colaborar para manter ou recuperar a saúde dos indivíduos e estimular hábitos alimentares saudáveis (COLARES; FREITAS, 2007). Além da preocupação com o alimento ofertado as UPRs devem atuar no que se refere à sustentabilidade, pois podem causar grande impacto ambiental (DIAS; OLIVEIRA, 2016).

Um dos principais problemas relacionados à sustentabilidade nessas unidades é a geração de resíduos, advindos especialmente das embalagens e de produtos químicos. Materiais como papéis, papelões, plásticos, vidros, latas e embalagens cartonadas dificilmente são separados de modo correto para reciclagem (WANG, 2012; GRAU, 2015). Ocorre também desperdício de água e contaminação devido ao uso dos produtos químicos, além do gasto exacerbado de energia (CALE; SPINELLI, 2008; VEIROS; PROENÇA, 2010).

Outro problema é o desaproveitamento de alimentos. Este pode ser procedente de sobras, que são alimentos produzidos e não distribuídos, de restos que são alimentos não consumidos e deixados pelo cliente (VAZ, 2006), de armazenamento incorreto, pré-preparo e preparo. Desperdiçar é sinônimo de falta de qualidade no serviço, por isso um planejamento adequado é fundamental, evita produções em excesso e consequentemente sobras (SILVÉRIO; OLTRAMARI, 2014).

O planejamento da estrutura física de uma UPR também é de extrema 
importância, pois contribui para o correto funcionamento, evitando problemas com relação aos custos, operacionalização, riscos de contaminação entre alimentos e acidentes de trabalho (ALEVATO; ARAÚJO, 2009; CAMPOS, IKEDA, SPINELLI, 2012).

A sustentabilidade ambiental na produção de refeições pode ser desenvolvida através de práticas que amenizem o impacto ao meio ambiente através do uso consumo consciente de água, redução da geração de resíduos, aumento da reciclagem, utilização de alimentos agroecológicos, capacitação de funcionários, entre outras (MARTINELLI, 2011). A adequação desses fatores é um desafio para os gestores do serviço de alimentação, que precisam conciliar esses aspectos ao controle dos custos e demais demandas e necessidades do serviço e dos clientes (SPINELLI, 2009).

Cabe ao nutricionista, profissional responsável pelo planejamento e direcionamento das atividades nas unidades, capacitar à equipe, realizar ações de conservação dos recursos e diminuição da geração de resíduos (HARMON; GERALD, 2007) na elaboração do cardápio, dar preferência por alimentos da safra e/ou alimentos produzidos de um modo que favoreça o meio ambiente, priorizar o fornecimento de gêneros com embalagens recicláveis e preferir equipamentos que consomem menos água e energia (CAMPOS; LEMOS, 2005; PREUSS, 2009).

Diante do exposto, o objetivo do presente estudo foi avaliar a sustentabilidade ambiental e a percepção do nutricionista a respeito do tema em Unidades Produtoras de Refeições de Clubes Paulistanos.

\section{METODOLOGIA}

Trata-se de um estudo de delineamento transversal, realizado em Unidades Produtoras de Refeições (UPRs) de Clubes localizados na cidade de São Paulo, SP, com amostra composta por estabelecimentos tipo "self-service", "à la carte" e por porcionamento. Foram considerados self-service, os locais que apresentaram "buffet" livre com refeição por peso; "à la carte", locais com cardápio pré-estabelecido; e locais com funcionários que serviam preparações à maneira de "buffet livre", como restaurante por porcionamento.

Para a coleta de dados, foram utilizados um "check list" e um questionário adaptados de um instrumento de pesquisa da Universidade do Porto (UPorto) elaborado por Nóbrega, Veiros e Rocha (2018).

O check list foi aplicado para caracterização das unidades estudadas (tipo de UPR, número médio de refeições produzidas por dia, número de preparações no buffet frio, número de pratos proteicos, número de pratos base, número de guarnições, número de sobremesas e presença de programas de sustentabilidade) e para 
obtenção de aspectos de instalação, equipamentos, infraestrutura/área física, consumo de água, eletricidade, gás, produtos químicos, resíduos, hortifrutícolas e satisfação do cliente. Esses aspectos foram pontuados, cada item pontuava de 1 a 3 , sendo 3 o melhor valor no que se refere a sustentabilidade. A pontuação total máxima era de 195 pontos ou 100\%. A aplicação do instrumento foi realizada por um estudante de Nutrição, mediante observação do local e informações cedidas pelo nutricionista.

Também foi aplicado um questionário cuja pontuação máxima é de 54 pontos ou $100 \%$ para avaliação da percepção pessoal do nutricionista com relação à sustentabilidade ambiental, em que cada questão pontuava de 1 a 3 , sendo 3 o melhor valor no que se refere à sustentabilidade. Nele, perguntou-se o tempo de atividade na profissão, formação, nível de escolaridade e questões referentes ao consumo de água, energia, geração de resíduos, qualidade dos produtos, satisfação do cliente e desperdício. A aplicação foi executada por meio de entrevista e foi estabelecido como critério que esta fosse realizada com o nutricionista responsável pela UPR.

Ambos os instrumentos são classificados em: "muito bom" quando a pontuação atingia de 90 a 100\%, "bom" de 75 a 89\%, "aceitável" de 50 a 74\% e "não aceitável" se menor que $49 \%$.

Os resultados foram analisados por meio da média e desvio padrão. Variáveis quantitativas foram testadas em relação à sua aderência a distribuição normal e, como todas resultaram em distribuição normal, foram utilizados testes estatísticos paramétricos. Utilizou-se o teste $t$ de Student para analisar a diferença entre duas médias e a análise de variância (ANOVA) para analisar a diferença entre três ou mais médias. As análises de associação entre duas variáveis quantitativas foram realizadas por meio da correlação de Pearson. Todos os testes estatísticos foram realizados no programa SPSS versão 21 e consideraram nível de significância de 5\%.

Em relação à ética em pesquisa, o presente estudo está aprovado pelo comitê de ética da Universidade Presbiteriana Mackenzie, como parte de estudo maior denominado Qualidade em Serviços de Alimentação e registrado sob CAAE 48483015.7.0000.0084. Os indivíduos que compuseram a amostra, bem como as instituições participantes, receberam um termo de consentimento livre e esclarecido para autorizar a sua participação.

\section{RESULTADOS E DISCUSSÃO}

A amostra foi composta por sete Unidades Produtoras de Refeições de sete clubes paulistanos, sendo $71,4 \%(n=5)$ do tipo "self service", $14,3 \%(n=1)$ "à la carte" e $14,3 \%(n=1)$ por porcionamento.

A média diária de refeições foi de 1221 , com desvio padrão de \pm 865 . A Tabela 1 apresenta a quantidade, média e desvio padrão das preparações servidas nas 
unidades estudadas.

Tabela 1 - Quantidade, média e desvio padrão de preparações servidas em Unidades Produtoras de Refeições (UPRs) de São Paulo, SP, 2018.

\begin{tabular}{|c|c|c|c|c|c|c|c|c|c|}
\hline UPR's & $A$ & $B$ & C & $\mathrm{D}$ & $E$ & $\mathrm{~F}$ & G & Média & DP \\
\hline $\begin{array}{l}\text { Preparações buffet } \\
\text { frio }\end{array}$ & 4 & 5 & 2 & 5 & 2 & 13 & 8 & 5,6 & $\pm 3,9$ \\
\hline Pratos proteicos & 2 & 3 & 2 & 3 & 3 & 3 & 8 & 3,4 & $\pm 2,1$ \\
\hline Pratos base & 3 & 3 & 3 & 3 & 6 & 4 & 3 & 3,6 & $\pm 1,1$ \\
\hline Guarnição & 2 & 2 & 1 & 2 & 2 & 2 & 4 & 2,1 & $\pm 0,9$ \\
\hline Sobremesa & 4 & 3 & 3 & 3 & 9 & 7 & 8 & 5,3 & $\pm 2,6$ \\
\hline $\begin{array}{l}\text { Total das pre } \\
\text { ofertadas }\end{array}$ & 15 & 16 & 11 & 16 & 22 & 29 & 31 & 20 & $\pm 6,6$ \\
\hline
\end{tabular}

Fonte: Os autores.

Do total de restaurantes, $71,4 \%(n=5)$ possuíam programas voltados para sustentabilidade ambiental. Em um estudo com restaurantes comerciais de São Paulo, dos 30 locais estudados nenhum declarou aplicar algum programa para gestão ambiental (BARTHICHOTO et al., 2013). Em outro estudo, feito com restaurantes de Florianópolis, não foi referida a existência de um programa voltado para redução de impacto ambiental, porém foi constatado que de 40 restaurantes, 82\% possuíam medidas/ações sustentáveis em relação ao meio ambiente (KREMER; COSTA; MONDO, 2013).

A gestão ambiental faz parte de um novo modelo de negócio, a Gastronomia Sustentável pode auxiliar na obtenção de resultados financeiros, diminuindo os impactos ambientais que seus processos produtivos causam, melhorando o lucro e a imagem da marca para os clientes (NUNES, 2012).

No presente estudo, foi possível observar que os restaurantes que dispunham de programas de sustentabilidade ambiental (UPR's A,B,C,D e E) apresentaram maior pontuação no check list. Esse fato pode indicar que o programa não só existe, como é aplicado.

Verificou-se (Quadro 1) que $85,7 \% \quad(n=6)$ unidades obtiveram pontuação correspondente a classificação "aceitável" e uma (14,3\%) foi classificada como "bom", ou seja atingiu de 75 a $89 \%$ no check list.

Quadro 1 - Pontuação obtida por tema e no total do "check list" aplicado nas Unidades Produtoras de Refeições (UPR's). São Paulo, 2018.

\begin{tabular}{|lccccccc|}
\hline & & \multicolumn{7}{c|}{ UPR's } \\
\hline Temas: & $\mathrm{A}$ & $\mathrm{B}$ & $\mathrm{C}$ & $\mathrm{D}$ & $\mathrm{E}$ & $\mathrm{F}$ & $\mathrm{G}$ \\
Infraestrutura/área física (\%) & $\mathbf{4 4 , 4}$ & $\mathbf{5 5 , 6}$ & $\mathbf{4 4 , 4}$ & $\mathbf{7 7 , 8}$ & $\mathbf{5 5 , 6}$ & $\mathbf{5 5 , 6}$ & $\mathbf{5 5 , 6}$ \\
Consumo de água (\%) & $\mathbf{8 2 , 4}$ & $\mathbf{8 4 , 3}$ & $\mathbf{7 0 , 6}$ & $\mathbf{7 6 , 5}$ & $\mathbf{7 0 , 6}$ & $\mathbf{6 2 , 7}$ & $\mathbf{5 2 , 9}$ \\
Consumo de eletricidade (\%) & $\mathbf{7 3 , 8}$ & $\mathbf{7 9 , 8}$ & $\mathbf{7 1 , 4}$ & $\mathbf{7 1 , 4}$ & $\mathbf{7 6 , 2}$ & $\mathbf{3 8 , 1}$ & $\mathbf{7 3 , 8}$ \\
Consumo de gás (\%) & $\mathbf{7 4 , 1}$ & $\mathbf{7 7 , 8}$ & $\mathbf{6 6 , 7}$ & $\mathbf{7 0 , 4}$ & $\mathbf{8 8 , 9}$ & $\mathbf{7 4 , 1}$ & $\mathbf{7 7 , 8}$
\end{tabular}




\begin{tabular}{|lccccccc|} 
Produtos químicos (\%) & $\mathbf{9 3 , 3}$ & $\mathbf{8 0}$ & $\mathbf{1 0 0}$ & $\mathbf{1 0 0}$ & $\mathbf{8 0}$ & $\mathbf{7 6 , 7}$ & $\mathbf{3 3 , 3}$ \\
Resíduos (\%) & $\mathbf{8 7 , 9}$ & $\mathbf{6 3 , 6}$ & $\mathbf{6 0 , 6}$ & $\mathbf{6 9 , 7}$ & $\mathbf{6 3 , 6}$ & $\mathbf{4 2 , 4}$ & $\mathbf{4 5 , 5}$ \\
Hortifrutícolas (\%) & $\mathbf{3 3 , 3}$ & $\mathbf{1 6 , 7}$ & $\mathbf{1 6 , 7}$ & $\mathbf{3 3 , 3}$ & $\mathbf{1 6 , 7}$ & $\mathbf{3 3 , 3}$ & $\mathbf{3 3 , 3}$ \\
Satisfação do cliente (\%) & $\mathbf{1 0 0}$ & $\mathbf{1 0 0}$ & $\mathbf{1 0 0}$ & $\mathbf{3 3 , 3}$ & $\mathbf{1 0 0}$ & $\mathbf{3 3 , 3}$ & $\mathbf{3 3 , 3}$ \\
\hline Pontuação geral (\%) & 77,4 & 74,6 & 67,7 & 72,1 & 72,3 & 53,6 & 59,5 \\
\hline
\end{tabular}

Fonte: Os autores.

A pontuação do "check list" analisada tipo de restaurante não variou estatisticamente (Tabela 2).

Tabela 2 - Média e desvio padrão da pontuação do "check list" segundo tipo de restaurante. São Paulo, 2018.

\begin{tabular}{ccccl}
\hline Tipo de restaurante & \multicolumn{4}{c}{ Pontuação do check list } \\
& $\mathrm{n}$ & Média & DP & Valor $\mathrm{p}$ \\
\hline 1 & 5 & 131,50 & 20,16 & 0,912 \\
2 & 1 & 132,00 & --- & \\
3 & 1 & 141,00 & --- & \\
\hline
\end{tabular}

Fonte: Os autores.

Ao analisar a associação entre o número de refeições e a pontuação do "check list", obteve-se correlação fraca $(r=0,523)$. A quantidade de refeições servidas e o tipo de restaurante não estiveram relacionados com uma melhor pontuação do "check list".

A infraestrutura/área física apresentou pontuações baixas na maioria das unidades, devido à presença de iluminação e ventilação artificial e ambientes muito fechados, fatores que exigem maior demanda de energia elétrica.

Com relação ao consumo de água, $57,1 \%(n=4)$ das unidades obtiveram a classificação "aceitável", o que possivelmente indica um elevado consumo, enquanto que $42,9 \%(n=3)$ foram classificadas como "bom", o que pode indicar um consumo mais controlado. Foi verificado se o local registrava ou não o consumo de água, se havia metas para o consumo, conservação de torneiras, chuveiro, descargas, água de reuso, descongelamento de alimentos entre outros aspectos.

A água é indispensável no fornecimento de refeições. Usada durante o preparo, nos processos de limpeza e higienização de alimentos, equipamentos, utensílios e ambiente (STRASBURG; JAHNO, 2017). Portanto, é indicado seu uso de forma racional (ABREU; SPINELLI; SOUZA, 2016).

No que se refere ao consumo de eletricidade, uma unidade $(14,3 \%)$ obteve classificação "não aceitável", 57,1\% (n=4) "aceitável" e 28,6\% (n=2) "bom". No estudo de Barthichoto et al. (2013) apenas 37,5\% ( $n=12)$ dos 32 estabelecimentos verificados realizavam a mensuração do consumo de energia elétrica, enquanto que no presente estudo $85,7 \%$ das unidades $(n=6)$ a realizavam. Apenas uma unidade $(14,3 \%)$ 
apresentou alguns equipamentos com selo de eficiência energética. A manutenção preventiva dos equipamentos é realizada em $57,1 \%(n=4)$ dos locais. A American Dietetic Association (ADA) orienta a escolha de equipamentos de melhor eficiência energética, manutenção preventiva e desenvolvimento de estratégias para a economia de energia (HARMON; GERALD, 2007).

Quanto ao consumo de gás, todas as unidades faziam o registro do consumo, $57,1 \%(n=4)$ utilizavam gás natural e 42,9\% $(n=3)$ gás liquefeito de petróleo (GLP). Segundo Ribeiro (2011), o uso do gás natural contribui para o aumento do ganho ambiental, pois ele pode ser descrito como um combustível de queima limpa, quando comparado a outros combustíveis fósseis altamente poluentes e capazes de causar danos irreversíveis ao meio ambiente.

Os produtos químicos de limpeza possuíam ficha técnica de utilização em $85,7 \%$ $(n=6)$ dos locais e as indicações presentes nas fichas eram respeitadas. Apenas um local $(14,3 \%)$ utilizava produtos com a declaração de biodegradável na embalagem. 0 uso de elevada quantidade de produtos de limpeza resulta em um elevado potencial de poluição, pois a mistura de vários compostos químicos com as sujidades, deixa resíduos nos efluentes (VÉRAS, 2002). Uma provável limitação para o baixo uso de biodegradáveis é o elevado custo. No estudo de Corrêa e Lange (2011), por exemplo, verificou-se que o requisito para seleção de produtos de limpeza e higienização era o preço.

Os resíduos sólidos eram separados em 57,1\% $(n=4)$ das unidades. Outro estudo com restaurantes comerciais na região da Granja Vianna verificou que dos 30 locais estudados $76,6 \%$ afirmaram realizar separação de resíduos segundo sua composição (FERNANDES; SPINELLI, 2016). Um número menor foi encontrado no estudo de Barthichoto et al. (2013) com a coleta seletiva de resíduos sólidos realizada por $25 \%$ dos locais $(n=8)$, resultado mais próximo do verificado no presente estudo.

Na maioria dos locais $(71,4 \%)(n=5)$ as sobras eram registradas. De acordo com Abreu, Spinelli e Souza (2016), o controle de sobras é um importante indicador de desperdício e está relacionado com problemas de planejamento. Esse desperdício implica na perda dos recursos que foram necessários para preparar os alimentos, inclusive água e energia (PIRANI; ARAFAT, 2014).

Todas as UPR's utilizavam matérias descartáveis em seu serviço. Sabe-se que o uso de descartáveis possibilita maior higiene e segurança, porém, no sentido de se evitar desperdício e excesso de descarte, é fundamental que sua utilização ocorra de forma racional (KINASZ; WERLE, 2006).

Os hortifrutícolas adquiridos em 100\% das UPR's provêm de produção não orgânica e em 57,1\% ( $n=4)$ vêm de centros de abastecimento. Nenhuma relatou aquisição de produtos originados da agricultura familiar. Em restaurantes comerciais de Pelotas, verificou-se que do total da amostra $(n=21)$ apenas $14,29 \% \quad(n=3)$ 
utilizavam alimentos de produtores locais/agricultura familiar no cardápio total e $47,62 \%$ ( $n=10)$ utilizava no cardápio parcial. Foi demonstrado interesse em utilizar produtos oriundos da agricultura familiar no cardápio total, mas a quantidade, de alimentos necessária, não conseguiria ser suprida por pequenos produtores (GONÇALVES et al., 2018), fato que possivelmente justifica a não utilização desses produtos também no presente estudo, visto que os locais estudados produzem elevadas quantidades de refeições diariamente.

A avaliação da satisfação do cliente era realizada em 57,1\% (n=4) da amostra. Segundo Ferraz (2016) há uma forte relação entre a satisfação do cliente e as práticas sustentáveis adotadas pelas unidades de alimentação e nutrição, uma vez que a insatisfação do cliente gera como consequência o desperdício de alimentos, provocando danos ao meio ambiente, sobrecarrega o serviço e gera gastos desnecessários de matérias-primas e recursos hídricos e energéticos. Apesar da evidente importância da análise da satisfação do cliente, não houve diferença estatisticamente significativa na pontuação média do "check list", segundo satisfação do cliente no presente estudo (Tabela 3).

Tabela 3 - Média e desvio padrão da pontuação do check list segundo satisfação do cliente. São Paulo, 2018.

\begin{tabular}{lllll}
\hline $\begin{array}{l}\text { Satisfação } \\
\text { cliente }\end{array}$ & do & \multicolumn{2}{l}{ Pontuação do check list } \\
& $\mathrm{n}$ & Média & DP & Valor $\mathrm{p}$ \\
\hline 1 & 3 & 120,33 & 18,39 & 0,080 \\
3 & 4 & 142,38 & 8,04 & \\
\hline
\end{tabular}

Fonte: Os autores.

Verifica-se que a percepção a respeito de sustentabilidade ambiental da maioria dos nutricionistas $(71,4 \%)$ é muito boa (Gráfico 2 ).

Gráfico 2 - Pontuação e classificação obtida pelo nutricionista de cada unidade no questionário de percepção de sustentabilidade ambiental, São Paulo, 2018.

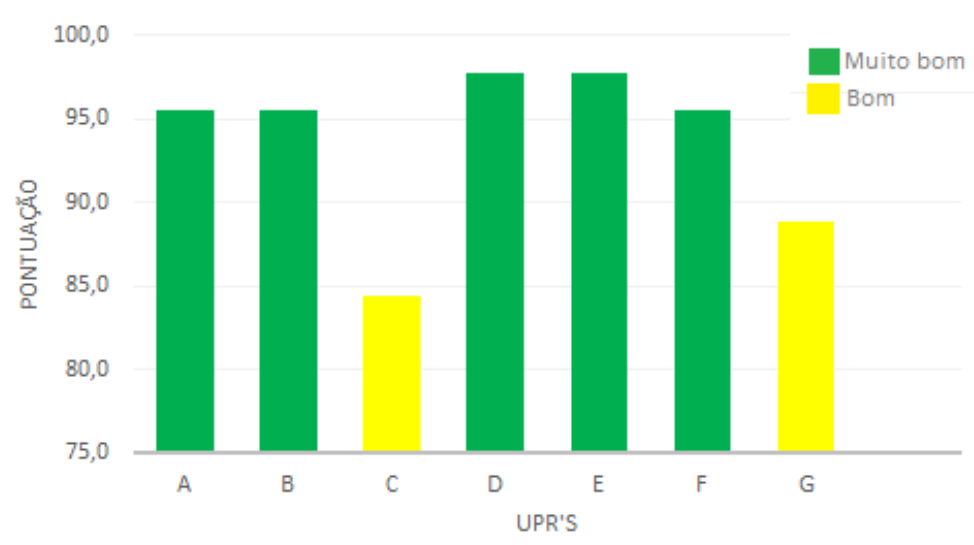

Fonte: Os autores. 
A análise da associação entre a percepção de sustentabilidade e a pontuação do "check list", resultou em um coeficiente de correlação de Person de 0,309, o que representa uma correlação fraca.

Todos os profissionais entrevistados $(n=7)$ eram nutricionistas graduados, sendo que $42,9 \%(n=3)$ possuíam nível superior completo e 42,9 $(n=3)$ eram pós-graduados. O tempo médio de atividade na profissão foi de aproximadamente 5 anos. Uma pessoa optou por não referir o nível de escolaridade e tempo médio de profissão. Ao analisar a associação entre o tempo de profissão e a pontuação do "check list", obteve-se correlação fraca $(r=0,525)$, o que pode indicar que as práticas de medidas de menor impacto ambiental não estão relacionadas com uma maior experiência e/ou maior tempo de atividade na profissão do nutricionista.

O presente estudo apresentou como limitação o tamanho da amostra. Outros locais foram convidados para participar, porém não manifestaram interesse e não são todos os clubes da capital que apresentam serviço de alimentação.

\section{CONCLUSÃO}

Foi possível verificar que a maioria dos nutricionistas possuem elevada percepção sobre sustentabilidade, porém ao observar as pontuações do "check list" percebe-se que são poucas as ações realizadas de modo a minimizar o impacto ambiental.

As principais medidas sustentáveis encontradas foram em relação ao consumo de água, observadas por meio do bom estado de conservação de torneiras, vasos sanitários, chuveiros e técnicas de descongelamento. Quanto ao consumo de energia, observou-se o bom estado de conservação de tomadas e equipamentos. Para os produtos químicos, verificou-se se as fichas técnicas eram respeitadas e se havia presença de dosadores. Também foi verificada se havia ou não a realização de reciclagem. A estrutura física (iluminação/ventilação), uso de descartáveis, controle e registro de sobras/resto e aquisição de hortifrutícolas (tipo de produção), são aspectos que deverão ser aprimorados.

A relevância deste estudo se deu no sentido de despertar a consciência dos profissionais, de demonstrar seu importante papel na implantação de ações sustentáveis em restaurantes, por meio do controle de resíduos, consumo consciente de água, gás e energia, redução do desperdício de alimentos, entre outras. Sugere-se que mais pesquisas sejam realizadas com esse tema, de modo a conscientizar os profissionais e incentivar a implementação de práticas sustentáveis no segmento de produção de refeições.

\section{REFERÊNCIAS}


ABREU, E. S.; SPINELLI, M. G. N. SOUZA, A. M. P. Gestão de unidades de alimentação e nutrição: um modo de fazer. 6. ed. São Paulo: editora Metha, 2016.

ALEVATO, H.; ARAÚJO, E.M.G. Gestão, organização e condições de trabalho. In: CONGRESSO NACIONAL DE EXCELÊNCIA EM GESTÃO, 5, 2009, Niterói. Anais..., 2009. Disponível em:

<http://www.excelenciaemgestao.org/Portals/2/documents/cneg5/anais/T8_0155_0577 .pdf>. Acesso em: 23 nov. 2017.

BARTHICHOTO, M. et al, Responsabilidade ambiental: perfil das práticas de sustentabilidade desenvolvidas em unidades produtoras de refeições do bairro de Higienópolis, município de São Paulo, Qualit@s Rev. Eletrônica, São Paulo, v. 14, n. 1, p. 1-9, 2013.

BEZERRA, I. N. et al. Consumo de alimentos fora do lar no Brasil segundo locais de aquisição. Revista de Saúde Pública, v. 51, n. 15, 2017.

CALE, L.; SPINELLI, M. G. N. Controle de Resíduos: Responsabilidade Social do Nutricionista. Revista Nutrição Profissional, São Paulo, n. 19, p. 32-38, 2008.

CAMPOS, I. C.; LEMOS, M. Implantação da ISO 14001 na unidade de alimentação e nutrição de uma indústria de Santa Catarina, Brasil: Preliminares. Revista Nutrição em Pauta, v. 13, n. 72, p. 30-35, 2005.

CAMPOS, J. R.; IKEDA, V.; SPINELLI, M. G. N. Otimização de espaço físico em Unidade de Alimentação e Nutrição (UAN) considerando avanços tecnológicos no segmento de equipamentos, Revista Univap, São José dos Campos, v. 18, n. 32, p. 31-41, 2012.

COLARES, L. G. T.; FREITAS, C. M. Processo de trabalho e saúde de trabalhadores de uma unidade de alimentação e nutrição: entre a prescrição e o real do trabalho.

Cad. Saúde Pública, Rio de Janeiro, v. 23, p. 3011-3020, 2007.

CORREAA, M. S.; LANGE, L. C. Gestão de resíduos sólidos no setor de refeição coletiva. Pretexto, v. 12, n. 1, 2011.

DIAS, N. A.; OLIVEIRA, A. L. Sustentabilidade nas unidades de alimentação e nutrição: desafios para o nutricionista no século XXI. Higiene Alimentar, Minas Gerais, v. 30, n. 254-255, p. 26-31, 2016.

DIEZ GARCIA, R.W. Reflexos da globalização na cultura alimentar: considerações sobre as mudanças na alimentação urbana. Rev. Nutr., Campinas, v. 16, n. 4, p. 483492, 2003.

FERNANDES, M. C.; SPINELLI, M. G. N. Análise de práticas sustentáveis em restaurantes comerciais na região da Granja Vianna-SP - práticas sustentáveis em restaurantes. In: JORNADA DE INICIAÇÃO CIENTÍFICA E VI MOSTRA DE INICIAÇÃO TECNOLÓGICA, 12, 2016. Anais..., São Paulo: Universidade Presbiteriana Mackenzie, 2016.

FERRAZ, T. V. Práticas sustentáveis em restaurantes universitários de universidades federais brasileiras. 2016. 93f. Tese (Mestrado Profissional em Gestão Pública) - Universidade Federal do Espírito Santo, Vitória, 2016.

GONÇALVES, et al. Sustentabilidade ambiental em restaurantes comerciais da zona 
central de Pelotas - RS. R. gest. sust. ambient., Florianópolis, v. 7, n. 2, p. 525-539, 2018.

GRAU - GREEN RESTAURANTS ASSOCIATION UNIVERSITY.

Green Restaurant Certification 4.0 Standards. 2015. Disponível em:

<http://www.dinegreen.com/restaurants/standards.asp>. Acesso em: 22 nov. 2017.

HARMON, A. H.; GERALD, B. L. Position of the American Dietetic Association: Food and Nutrition Professionals Canlmplement Practices to Conserve Natural Resources and Support Ecological Sustainability. Journal of the American Dietetic Association, v. 107, n. 6, p. 1033-1043, 2007.

IBGE. Instituto Brasileiro de Geografia e Estatística. Pesquisa de Orçamentos Familiares 2008-2009: Análise do Consumo Alimentar Pessoal no Brasil. Rio de Janeiro, 2011. Disponível em:

<https://biblioteca.ibge.gov.br/visualizacao/livros/liv50063.pdf>. Acesso em: 10 abr. 2019.

KINASZ, T. R.; WERLE, H. J. S. Produção e composição física de resíduos sólidos em alguns serviços de alimentação e nutrição, nos municípios de Cuiabá e Várzea Grande, Mato Grosso: questões ambientais. Hig. Aliment., v. 20, n. 44, p. 64-71, 2006.

KREMER, E. C.; COSTA, J. I. P; MONDO, T. S. análise das ações de responsabilidade ambiental implementadas nos restaurantes do centro de Florianópolis. TURyDes, v. 6, n. 14, 2013.

MARTINELLI, S. S. Desenvolvimento de método de qualidade nutricional, sensorial, regulamentar e sustentabilidade no abastecimento de carnes em unidades produtoras de refeições: o exemplo da carne bovina. 2011. Dissertação (Mestrado em Nutrição) - Universidade Federal de Santa Catarina, Florianópolis, 2011.

NÓBREGA, F.; VEIROS, M.; ROCHA, A. Aspectos ambientais em unidades de alimentação coletiva - o caso dos serviços de ação social da Universidade do Porto. In: CONGRESSO DE NUTRIÇÃO E ALIMENTAÇÃO, 17, 2018, Lisboa. Anais..., Lisboa: Acta Portuguesa de Nutrição; 2018.

NUNES, R. Gastronomia Sustentável. Interação - Revista Científica da Faculdade das Américas, São Paulo, ano III, n. 1, jan./jul. 2012.

PIRANI, S.I.; ARAFAT, H.A. Solid waste management in the hospitality industry: a review. Journal of Environmental Management, v. 146, p. 320-336, 2014.

PREUSS, K. Integrando nutrição e desenvolvimento sustentável: atribuições e ações do nutricionista. Nutr. Pauta,v. 17, n. 99, p. 50-53, 2009.

RIBEIRO, R. N. A geopolítica de distribuição de gás natural Europeia / Asiática e a Rússia como grande protagonista deste cenário. 2011. Trabalho de Conclusão de Curso (Graduação em Engenharia do Petróleo) - Universidade Federal do Rio de Janeiro, Rio de Janeiro, 2011.

RODGERS, S. Food service research: An integrated approach. International Journal of Hospitality Management, v. 30, n. 2, p. 477-483, 2011.

SILVÉRIO, G.A.; OLTRAMARI, K. Desperdício de alimentos em Unidades de 
Alimentação e Nutrição brasileiras. Rev. do Setor de Ciências Agrárias e Ambientais, Guarapuava, v. 1, n. 10, p. 125-33, 2014.

SPINELLI, M. G. N. Gestão adequada dos serviços pode gerar aumento da deficiência operacional e diminuir despesas, beneficiando o meio ambiente. Revista do Conselho Regional de Nutricionistas- CRN 3 ${ }^{\text {a }}$ região, 2009.

STRASBURG, V. J.; JAHNO, V. D. Paradigmas das práticas de gestão ambiental no segmento de produção de refeições no Brasil. Eng Sanit Ambient, v. 22 n. 1, p. 3-12, 2017.

VAZ, C.S. Restaurantes: controlando custos e aumentando lucros. Brasília: Metha. 193 p, 2006.

VEIROS, M. B.; PROENÇA, R. P. C. Princípios de Sustentabilidade na Produção de Refeições. Rev. Nutrição em Pauta, p. 45-49, 2010.

VÉRAS, A. Impacto ambiental da indústria de alimentos. In: GERMER, S. P. M. A indústria de alimentos e o meio ambiente. Campinas: ITAL, p. 60-61, 2002.

WANG, R. Investigations of Important and Effective Effects of Green Practices in Restaurants. Procedia - Social and Behavioral Sciences, v. 40, p. 94-98, 2012. 